\title{
Hindsight is 20/20: Reflections on an elusive diagnosis
}

\author{
Arman Kilic, MD
}

\author{
From the Division of Cardiac Surgery, University of Pittsburgh Medical Center, Pittsburgh, Pa. \\ Disclosures: Author has nothing to disclose with regard to commercial support. \\ Received for publication Sept 18, 2018; accepted for publication Sept 20, 2018 \\ Address for reprints: Arman Kilic, MD, University of Pittsburgh Medical Center, 200 Lothrop St, Suite C-700, \\ Pittsburgh, PA 15213 (E-mail: kilica2@upmc.edu). \\ J Thorac Cardiovasc Surg 2019;157:e15 \\ 0022-5223/\$36.00 \\ Copyright (C) 2018 Published by Elsevier Inc. on behalf of The American Association for Thoracic Surgery \\ https://doi.org/10.1016/j.jtcvs.2018.09.093
}

In this issue of the Journal, Hamuro and colleagues ${ }^{1}$ present an interesting report in which a membranous ventricular septal defect (VSD) with an aneurysmal septal leaflet of the tricuspid valve was misdiagnosed as an aneurysm of the membranous septum. This confusion in diagnosis has been reported as early as the 1960s. Chesler and colleagues ${ }^{2}$ described 5 cases in which anomalies of the tricuspid valve resembled aneurysms of the membranous septum. Moreover, there were 3 cases in which a VSD was associated with a tricuspid leaflet pouch, and 2 cases in which anomalous attachment of the septal leaflet of the tricuspid valve to the edge of a VSD resulted in a pouchlike structure. A subsequent study published in the 1970s reviewed 118 patients with endocardial cushion defects, and tricuspid pouch was diagnosed in 15 of these patients. ${ }^{3}$ Pouches appeared similar to membranous septal aneurysms, although an important distinction was that most pouches protruded into the inflow portion of the right ventricle, whereas most membranous septal aneurysms protruded to the outflow portion of the right ventricle.

The images provided in the report by Hamuro and colleagues ${ }^{1}$ are informative, highlighting a large membranous VSD that was missed during the initial operation. This misdiagnosis led to an early reoperation and closure of the VSD on postoperative day 21. The absence of a shunt during the index hospitalization likely clouded the diagnosis. So, what clues can surgeons use in the future to identify this elusive diagnosis? As suggested by Mavroudis and Backer $^{4}$ in an article focusing on operative techniques, membranous defects are often covered by the tricuspid septal leaflet and may be difficult to visualize. A clue to the VSD may be depression in the tricuspid valve caused by valve tissue being sucked down into the defect, particularly in cases in which the chordal attachments of the tricuspid valve to the edge of the defect are short, or there is aneurysmal or redundant tricuspid leaflet tissue. As was done during the reoperation in the report of Hamuro and cases.

\section{References} diol. 1968;21:661-8.

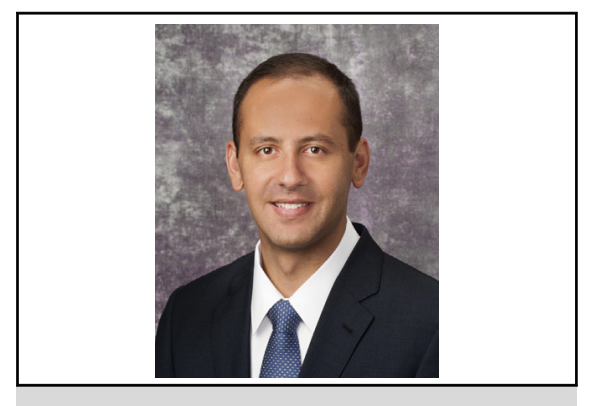

Arman Kilic, MD

\section{Central Message}

This is an interesting report in which a membranous ventricular septal defect with an aneurysmal septal leaflet of the tricuspid valve was misdiagnosed as an aneurysm of the membranous septum.

See Article page e11.

colleagues, ${ }^{1}$ detachment of the tricuspid leaflet often will provide necessary exposure to appreciate the underlying VSD and treat it surgically.

Placement of a rigid tricuspid ring that was abutting a thinned out and aneurysmal septal leaflet of the tricuspid valve that was subject to systemic pressures predictably led to erosion and creation of a shunt in this case. In a way, this was fortunate in that it led Hamuro and colleagues ${ }^{1}$ to the correct diagnosis with appropriate surgical revision and treatment early after the initial operation. Hindsight is certainly 20/20, and the authors ${ }^{1}$ provide an important case report detailing how these various diagnostic entities are easily confused, as corroborated by previous reports as well, underscoring the importance of careful preoperative and intraoperative anatomic evaluation in these

1. Hamuro M, Setozaki S, Enomoto S, Ikai A. Perforation of tricuspid pouch after tricuspid ring annuloplasty. J Thorac Cardiovasc Surg. 2019;157:e11-3.

2. Chesler E, Korns ME, Edwards JE. Anomalies of the tricuspid valve, including pouches, resembling aneurysms of the membranous ventricular septum. Am J Car-

3. Kudo T, Yokoyama M, Imai Y, Konno S, Sakakibara S. The tricuspid pouch in endocardial cushion defect. Am Heart J. 1974;87:544-9.

4. Mavroudis C, Backer CL. Closure of ventricular septal defect. Oper Tech Thorac Cardiovasc Surg. 2002; 7:11-21. 